BRITISH MEDICAL JOURNAL VOLUME $293 \quad 6$ SEPTEMBER 1986

A skull fracture is associated with an intracranial mass lesion in three quarters of patients. ${ }^{3}$ Absence of a fracture does not exclude serious head injury: $35 \%$ of patients in coma and $20 \%$ of those dying after head injury do not have a skull fracture. ${ }^{3}$ Computed tomography is mandatory for head injured patients in coma (Glasgow coma scale 8), and measurement of intracranial pressure may be useful. Half of head injured patients admitted in coma have a raised intracranial pressure, and intracranial pressure is raised in $30 \%$ of cases without haematoma. ${ }^{4}$ Raised intracranial pressure is also a feature of severe near drowning, reflecting the primary hypoxic insult. When the intracranial pressure is greater than $20 \mathrm{~mm} \mathrm{Hg}$ after near drowning outcome is poor (death in $70 \%$ of patients, a vegetative state in $30 \%)$. $^{5}$

In our patient attributing the neurological signs to severe head injury led to unnecessary monitoring of intracranial pressure and might have led inexperienced staff to diagnose post-traumatic brain stem failure and withdraw supportive treatment. When examining unconscious patients the core temperature should be measured and clinical signs interpreted accordingly.

1 Martin TG. Near drowning and cold water immersion. Ann Emerg Med 1984;13:263-73. 2 Fishbeck KH, Simon RP. Neurological manifestations of accidental hypothermia. Ann Neurol 1981;10:384-7.

3 Jennett B, Teasdale GM. Management of head injuries. Philadelphia: F A Davis, 1981

4 Miller JD, Butterworth JF, Gudeman SK, et al. Further experiences in management of severe head injury. I Neurosurg 1981;54:289-99.

5 Nussbaum E, Galant SP. Intracranial pressure monitoring as a guide to prognosis in the nearly drowned, severely comatose child. $\mathcal{F}$ Pediatr 1983;102:215-8.

(Accepted 25 fune 1986)

Western General Hospital, Edinburgh EH4 2XU

N V TODD, FRCs, registrar in surgical neurology

A CONN, FFARCS, senior registrar in anaesthetics.

Correspondence to: $\mathrm{Mr}$ Todd.

\section{Timing of disc surgery after iohexol myelography and its effect on the incidence of postoperative problems}

A recent study by Lynch and Dickson showed a significantly higher incidence of postoperative headache and nausea in patients undergoing discectomy within $\mathbf{4 8}$ hours of metrizamide myelography than in those whose operation was delayed for at least a week. ${ }^{1}$ Iohexol causes fewer adverse reactions after myelography than metrizamide, ${ }^{2}$ and we believed that this also applied after surgery. We therefore investigated the effect of the time interval between myelography and surgery on the incidence of postoperative problems in patients in whom iohexol had been used as the contrast medium.

\section{Patients and methods}

Thirty eight consecutive patients with low back pain and sciatica due to lumbar intervertebral disc prolapse who failed to respond to conservative measures were studied prospectively. Myelography was carried out using iohexol. After the procedure the patients were kept sitting up for six hours and remained on bed rest for at least a further 18 hours (if not operated on within that time).

The disc was subsequently excised through an interlaminar approach with the patient in the knee-elbow position. After the operation the presence or absence of headache, nausea, and urinary retention was recorded each day until the patient was discharged. The timing of the operation was determined solely by the availability of theatre time.

\section{Results}

The table shows the number of patients complaining of headache, nausea, and urinary retention on the first day after operation. The results were analysed with the exact probability test using different definitions of the early and late operations.

More patients operated on early had headaches on the first day after operation, but this difference was significant only when early operations were defined in one or other of two ways - namely, those performed on the day after the myelogram $(p=0.027)$ or those performed during the first three days after the myelogram $(p=0.046)$.

The number of patients with headaches after operation in the early operation group was significantly greater $(p=0.042)$ than the number with headaches after myelography alone (who were awaiting surgery), which suggested that the increased incidence was due to the operation and not simply to the after effects of the myelogram.

\section{Comment}

Adverse reactions after myelography are significantly reduced when iohexol rather than metrizamide is used as the contrast medium. ${ }^{2}$ If the contrast study is to be implicated in the production of symptoms in the postoperative period, as suggested by Lynch and Dickson, ${ }^{1}$ then the incidence of such symptoms should be reduced by using iohexol in preference to metrizamide. Furthermore, the timing of operations after myelography may be less important in preventing postoperative problems.

Incidence of headache, nausea, and urinary retention on the first day after operation

\begin{tabular}{cccccc}
\hline \multirow{2}{*}{$\begin{array}{c}\text { No of days between } \\
\text { myelogram and operation }\end{array}$} & $\begin{array}{c}\text { Total } \\
\text { No of } \\
\text { patients }\end{array}$ & \multicolumn{3}{c}{ No of patients with } \\
\cline { 3 - 5 } \cline { 4 - 5 } & & Headache & Nausea & Urinary retention \\
\hline 1 & 7 & 4 & 2 & 0 \\
2 & 4 & 0 & 2 & 0 \\
3 & 1 & 1 & 1 & 1 \\
4 & 3 & 0 & 0 & 1 \\
5 & 5 & 1 & 0 & 0 \\
6 & 4 & 0 & 0 & 2 \\
7 & 14 & 1 & 6 & \\
\hline
\end{tabular}

Our results show that the only significant difference between patients operated on early and those operated on late was in the incidence of headache on the first day after operation. The headache was not severe in any of the patients, and we do not believe that there is sufficient indication to delay surgery if theatre time is available soon after myelography has confirmed the diagnosis. Such a delay would merely increase the length of time the patient has to suffer sciatic pain.

1 Lynch AF, Dickson RA. The relationship of complications to the time between myelography and discectomy. I Bone foint Surg 1983;65B:259-61.

2 Kendall B, Schneidau A, Stevens J, Harrison M. Clinical trial of iohexol for lumbar myelography. Brf Radiol 1983;56:539-54.

(Accepted 1 fuly 1986)

Stafford District General Hospital, Stafford ST16 3SA

D RILEY, FRCS, orthopaedic registrar

J E WOODYARD, FRCS, consultant orthopaedic surgeon

H S DABIS, FRCS, orthopaedic registrar

Correspondence to: Mr D Riley, Orthopaedic Department, King's College Hospital, Denmark Hill, London SE5 9RS.

\section{Ceruletide for retained biliary stones}

The incidence of retained biliary stones after exploration of the common bile duct has been reported as $4 \cdot 3 \% .^{1}$ Initial therapeutic options include conservative management, perfusion techniques using the $T$ tube, and mechanical means. The use of saline alone to flush out small stones may be successful. Solvents such as cholate or mono-octanoate are more reliable but may have serious side effects. ${ }^{2}$ Stones may be extracted through the matured T tube tract or at endoscopic sphincterotomy, which has been reported to be successful in $85 \%$ of patients but is not universally available, may be technically difficult, and has potentially serious side effects. ${ }^{3}$

We describe our experience of a new perfusion technique that has previously been used successfully in four patients. ${ }^{4}$ Intravenous ceruletide exerts a cholecystokinetic action on the sphincter of Oddi, promoting relaxation and allowing increased volumes of saline to be infused through the $\mathrm{T}$ tube to flush out retained stones.

\section{Patients, methods, and results}

We have used this technique on 11 occasions in 10 patients over a year (table). All patients except one had undergone cholecystectomy and exploration of the 